\title{
Presencia y Morfometría de Forámenes y Canales Linguales en Cone Beam CT de Mandíbulas de Individuos Chilenos. Correlación con Medición Directa en Hueso
}

\author{
Presence and Morphometry of Lingual Foramina and Canal in Cone Beam CT \\ of Chilean Dry Mandibles. Correlation with Direct Bone Measures
}

Felipe Cáceres ${ }^{1}$; Sebastián Pardo $^{2}$; Guillermo Concha ${ }^{3} \&$ Reinaldo Soto $^{1}$

CÁCERES, F.; PARDO, S.; CONCHA, G. \& SOTO, R. Presencia y morfometría de forámenes y canales linguales en Cone Beam CT de mandíbulas de individuos chilenos. Correlación con medición directa en hueso. Int. J. Morphol., 35(1):299-305, 2017.

RESUMEN: Se han descrito forámenes ubicados en la cara interna del sector mandibular anterior, ya sea en hueso seco, radiografía convencional y en estudios mediante cone beam CT (CBCT). El objetivo de este estudio fue comparar la presencia, posición y las características anatómicas de los forámenes y canales linguales encontrados en CBCT de mandíbulas Chilenas secas, con los resultados de la misma muestra estudiada con visión directa en hueso seco. Se utilizaron 68 mandíbulas secas de la unidad de morfología de la Universidad de los Andes. Se evaluó número de dientes remanentes, número de forámenes relacionados con las espinas mentonianas, posición, área y longitud de cada canal en hueso seco y en CBCT de la misma muestra. Se encontró presencia del foramen lingual en 100 \% de CBCT y 97 \% de observación directa en hueso. Se observaron 150 forámenes en hueso seco y 170 forámenes en CBCT. La mayor cantidad se encontró por superior (hueso: 55; CBCT:61) e inferior (hueso:66 ; CBCT:92) a las espinas mentonianas. La confiablidad no fue la esperada, con un kappa para el número de forámenes superiores de 0,43, una correlación intraclase (rho) para el número de forámenes inferiores de 0,31. El área de forámenes superiores tuvo un rho de 0,059 e inferiores un rho de 0,144, mientras que, la longitud de los canales superiores, un rho de 0,5 e inferiores un rho de 0,246. Se observó una tendencia a mayores diámetros y longitudes de canales en las mediciones realizadas en CBCT comparadas con las mediciones en hueso seco. Teniendo en cuenta la alta prevalencia de estos forámenes, es recomendable previo a cualquier cirugía en el sector mandibular anterior, el estudio mediante CBCT. Además de mantener un margen de seguridad respecto a los forámenes linguales debido a una mínima distorsión esperable de estas estructuras.

PALABRAS CLAVE: Foramen lingual; Canal lingual, Cone beam CT; CBCT.

\section{INTRODUCCIÓN}

El conocimiento de la anatomía maxilar y mandibular es fundamental para el procedimiento quirúrgico en estas áreas. Se ha descrito la normalidad y variaciones anatómicas para ambos huesos en distintas poblaciones (Cova et al. 2003; Jacobs et al. 2002; Katakami et al. 2009; Kawashima et al. 2015; Patil et al. 2012; Zhang et al. 2015). Este artículo se enfoca en la presencia y características del foramen lingual ubicado en la línea mediana, descrito en la tabla lingual del sector mandibular anterior. Este foramen ha sido pobremente descrito en la literatura anatómica clásica, pero con el desarrollo de procedimientos quirúrgicos asociados a implantes dentales en el sector mandibular anterior, ha existido un aumento en las publicaciones sobre complicaciones asociadas con este elemento anatómico. Una de las complicaciones más peligrosa está relacionada con la hemorragia causada por la lesión de vasos asociados al foramen y canal lingual, los cuales pueden causar hematomas, incluso comprometiendo la vía aérea (Isaacson 2004; Kalpidis \& Setayesh 2004; Kilic et al. 2014). Teniendo estas complicaciones en consideración, se ha descrito la presencia y morfología de estos forámenes y canales en diferentes poblaciones, sexos y razas (Cáceres et al. 2014; Flanagan 2003; Liang et al. 2004; Liang et al. 2006; McDonnell et al. 1994; Soto et al. 2012) mostrando que los principales elementos que viajan por estos forámenes y canales son de características arteriales, con un flujo sanguíneo de manera centrífuga, desde la tabla lingual hacia la profundidad de la sínfisis mentoniana ya sea desde vasos sublinguales o submentonianos (Lustig et al. 2003; Romanos et al. 2012).

\footnotetext{
${ }^{1}$ Unidad de Morfología, Facultad de Odontología, Universidad de los Andes, Santiago, Chile.

${ }^{2}$ Especialista en Radiología Oral y Maxilofacial, Práctica Privada, Santiago, Chile.

${ }^{3}$ Departamento de Radiología Oral y Maxilofacial, Facultad de Odontología, Universidad de los Andes, Santiago, Chile.
} 
Por otro lado, la introducción del Cone Beam CT (CBCT) como herramienta de estudio imagenológico en el territorio maxilofacial, ha permitido una serie de ventajas, tales como, un mayor acceso a exámenes, menor costo económico y menor dosis de radiación (Mozzo et al. 1998). Además se ha demostrado su alta correlación con la forma y dimensiones del objeto estudiado (Kosalagood et al. 2014). Sin embargo, no se ha encontrado en la literatura artículos que estudien el desempeño del CBCT en la búsqueda y caracterización del foramen lingual, comparándolo con la visión directa del objeto a ojo desnudo.

El objetivo de este estudio fue comparar la presencia, posición y las características anatómicas (longitud y área) de los forámenes y canales linguales encontrados en CBCT de mandíbulas secas, de individuos chilenos, con los resultados de la misma muestra estudiada con visión directa en hueso seco (Cáceres et al. ; Soto et al.).

\section{MATERIAL Y MÉTODO}

Se estudió una muestra de 68 mandíbulas secas, obtenidas de la Unidad de Morfología de la Universidad de los Andes, Santiago, Chile, con sexo y edad indeterminados. Las mandíbulas fueron identificadas utilizando los números asignados en las publicaciones previas realizadas por este equipo (Cáceres et al.; Soto et al.). Luego, éstas fueron escaneadas utilizando un Cone Beam CT (CBCT) (ORTHOPHOS XG 3D®, Sirona Dental GmbH, Salzburg, Österreich), siendo etiquetadas con el número utilizado previamente en hueso seco. Los CBCT fueron tomados en alta resolución, con un voxel correspondiente a $160 \mu \mathrm{m}$. Un radiólogo experto fue entrenado para la medición, utilizando el coeficiente de correlación intraclase, mediante mediciones repetidas en el tiempo (mediciones en 10 mandíbulas de las distintas variables, separadas por 2 semanas). Una vez entrenado, se utilizó el software Sidexis XG® (Sirona Dental GmbH, Salzburg, Österreich) y se tabuló en una tabla Excel® las siguientes variables: Número de dientes remanentes en cada mandíbula, número de forámenes relacionados con las espinas mentonianas, posición de estos forámenes (superior a espinas mentonianas superiores (SEMS), entre espinas mentonianas superiores (EEMS), entre espinas mentonianas superiores e inferiores (EEMSI), entre espinas mentonianas inferiores (EEMI), inferior a espinas mentonianas inferiores (IEMI)) identificando la cantidad de forámenes en cada posición. Para medir el área para cada uno de estos forámenes, se utilizó un screenshot en el software Sidexis XG® (Sirona Dental GmbH, Salzburg, Österreich) a nivel de la entrada al canal de manera ortográfica, con la regla de referencia otorgada por el software (Fig
1). Luego utilizando el software Photoshop CS6 Extendend ® (Adobe Systems Incorporated, San José, California) se estudió la imagen, compensando los pixeles a mm con la referencia de la regla. Finalmente con la función Magic Wand Tool, se seleccionó el área del foramen, para luego con Record Measurements, obtener el área en milímetros cuadrados (Fig. 2). Para obtener la longitud de los canales se utilizó el software Sidexis XG®, el cual permite realizar mediciones lineales. En el caso que el canal se bifurcase dentro de la mandíbula, se consideró la longitud más larga (Fig. 3). Para la identificación de cada área y longitud respecto a su foramen y canal, se consideró en la posición en que se ubicaba respecto a las espinas mentonianas. Si existía más de un foramen en la posición, se nombraría de manera consecutiva desde la letra "a", considerando "a" como el más superior de los forámenes. Para la identificación de los forámenes y canales, junto con sus características morfométricas en el hueso seco, se utilizó la metodología y resultados publicados previamente por este equipo (Soto et al.), utilizando la misma clasificación de los forámenes respecto a las espinas mentonianas.

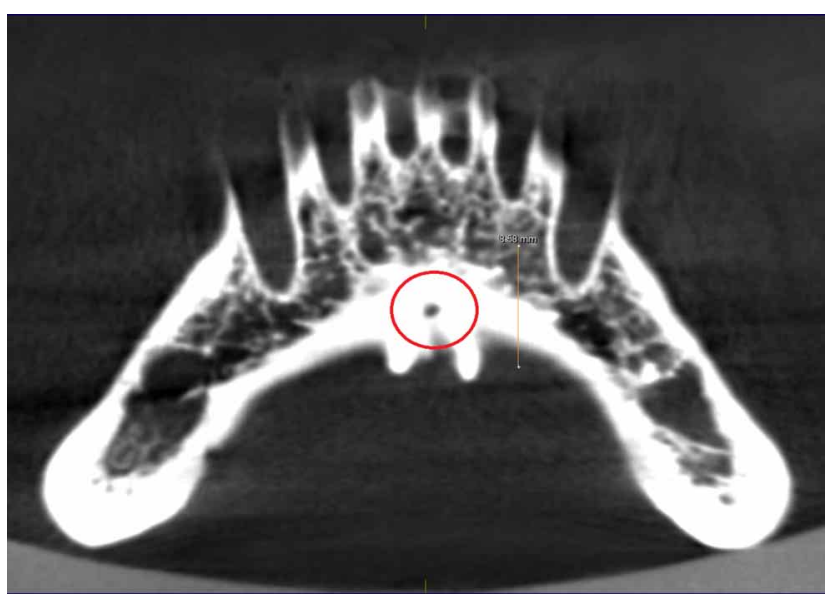

Fig. 1. Identificación del foramen lingual al inicio del canal, proyección ortográfica.

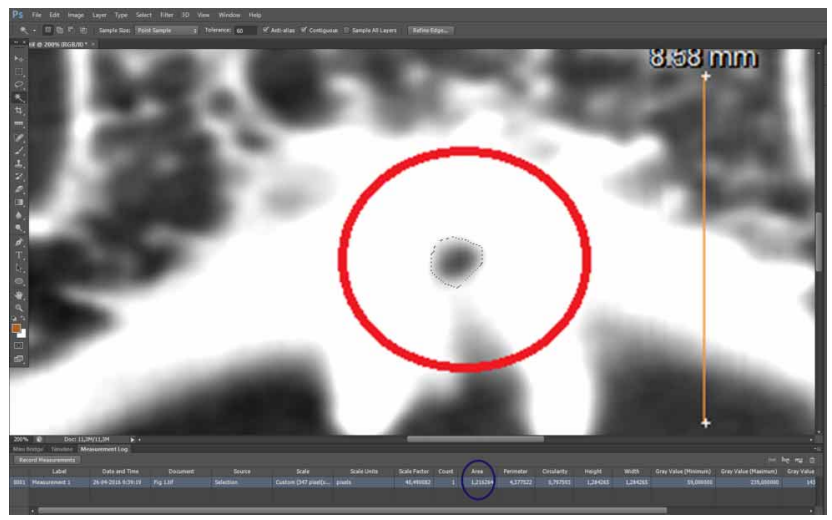

Fig. 2. Metodología de medición, luego de convertir pixeles en milímetros, utilizando "Magic Wand Tool" se seleccionó el área del foramen (línea punteada) para la obtención en $\mathrm{mm}^{2}$ del área. 


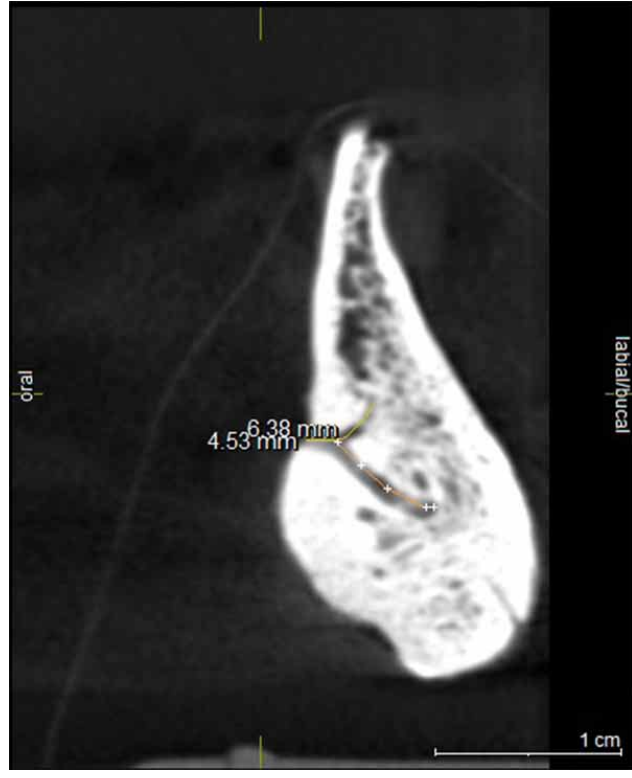

Fig. 3. Medición de canal lingual bifurcado.

Finalmente se realizó un test de correlación intraclase (variables continuas) e índice kappa (variables dicotómicas), para comparar los resultados obtenidos en el CBCT con los resultados en hueso seco publicados previamente de esta muestra. Las variables comparadas fueron número de piezas remanentes, número de forámenes, número de forámenes por posición, área de los forámenes y longitud de los forámenes.

Para obtener la estadística descriptiva y analítica de la muestra, se utilizó el software STATA LP 14® (StataCorp, College Station, Texas)

\section{RESULTADOS}

La descripción de la muestra evaluada en Cone Beam CT y en hueso seco se resume en la Tabla I. Se estudiaron 68 mandíbulas de sexo y edad indeterminada, encontrándose diferencias en la totalidad de las variables descritas.

Para la medición de las áreas de los forámenes y las longitudes de los canales que se desprendían de estos en CBCT, se realizó una calibración previa intraoperador, mediante coeficiente de correlación, siendo para el área un rho de 0,94 y para la longitud un rho de 0,93 en 10 mandíbulas medidas seguidas. La metodología de medición de las variables descritas en hueso seco, se encuentra disponible en publicaciones previas (Cáceres et al.; Soto et al.).

La descripción del área encontrada tanto en CBCT como en hueso seco se muestran en la Tabla II, mientras que la descripción de las longitudes de los canales que se desprenden de estos forámenes se puede observar en la Tabla III.

Se encontró un alto porcentaje de forámenes, teniendo el 97 \% de las mandíbulas analizadas directamente en hueso y el $100 \%$ de las mandíbulas estudiadas en CBCT, al menos un foramen lingual. Se observó una mayor cantidad de forámenes en los CBTC (170 forámenes) que el hueso seco, donde se encontraron una menor cantidad (150 forámenes).

En ambas exploraciones (ya sea hueso seco o CBCT) se encontró una importante cantidad de forámenes ubicados en las siguientes 3 posiciones: SEMS (hueso:55; CBCT: 61), EEMSI (hueso:17; CBCT:15) e IEMI (hueso:66: CBCT 92). En las posiciones más frecuentes, SEMS e IEMI, la cantidad de forámenes encontrados en CBCT, fue superior a los encontrados en visión directa de hueso. Para ambas exploraciones, se encontró un máximo de 1 foramen en la posiciones SEMS, EEMS, EEMSI, EEMI, en cambio para la posición IEMI, se encontraron un máximo de tres forámenes, nombrados consecutivamente IEMIa, IEMIb, IEMIc (Tablas II-III).

Respecto a la confiabilidad encontrada entre las medidas de CBCT y las directas en hueso, se encontró que para la variable "número de dientes remanentes" existió una alta confiabilidad (rho: 0,98), mientras que para "número total de forámenes" la confiabilidad fue menor (rho: 0,57). Para el resto de las variables, existió un grado variable de concordancia, considerándose una

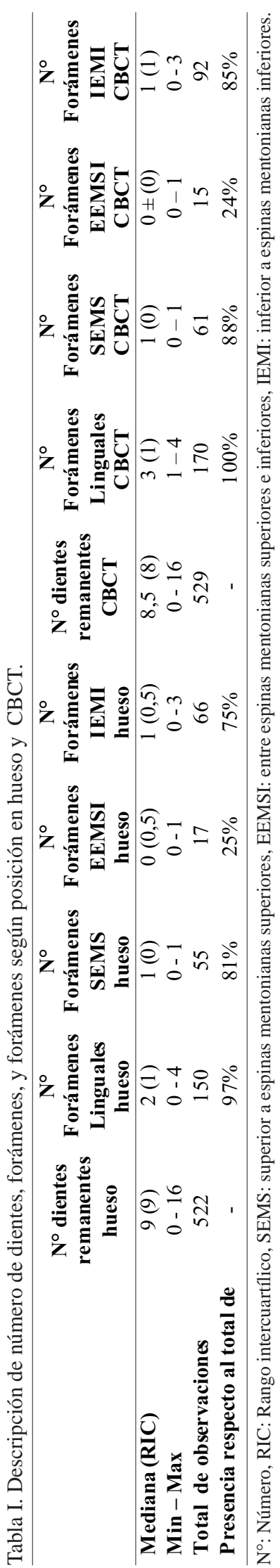


confiabilidad excelente, valores de rho sobre 0,75 o un kappa sobre 0,8 (Prieto et al. 1998) (Cerda \& Villarroel, 2008).
Los distintos grados de confiabilidad para las variables estudiadas se observan en la Tabla IV.

Tabla II. Áreas y números de forámenes según ubicación en hueso y CBCT.

\begin{tabular}{|c|c|c|c|c|c|c|}
\hline $\begin{array}{l}\text { Área estudiada } \\
\text { según ubicación }\end{array}$ & $\begin{array}{c}\text { Promedio en } \\
\text { mm }^{2} \text { Kueso }\end{array}$ & $\begin{array}{c}\text { Desviación } \\
\text { estándar hueso }\end{array}$ & $\begin{array}{c}\mathrm{N}^{\circ} \mathrm{de} \\
\text { forámenes en } \\
\text { cada posición } \\
\text { hueso }\end{array}$ & $\begin{array}{l}\text { Promedio en } \\
\text { mm }^{2} \& B C T\end{array}$ & $\begin{array}{c}\text { Desviación } \\
\text { estándar CBCT }\end{array}$ & $\begin{array}{l}\mathrm{N}^{\circ} \text { forámenes } \\
\text { en cada } \\
\text { posición CBCT }\end{array}$ \\
\hline SEMS & 0,47 & 0,33 & 55 & 0,96 & 0,76 & 61 \\
\hline EEMS & 0,37 & 0,04 & 4 & 2,12 & 1,41 & 2 \\
\hline EEMSI & 0,44 & 0,37 & 17 & 1,01 & 1,09 & 15 \\
\hline EEMI & 0,4 & 0,37 & 8 & & & 0 \\
\hline IEMIa & 0,53 & 0,42 & 51 & 0,87 & 0,61 & 61 \\
\hline IEMIb & 0,33 & 0,28 & 14 & 0,79 & 0,53 & 28 \\
\hline IEMIc & 0,22 & & 1 & 0,92 & 0,41 & 3 \\
\hline
\end{tabular}

SEMS: superior a espinas mentonianas superiores. EEMS: entre espinas mentonianas superiores. EEMSI: entre espinas mentonianas superiores e inferiores. EEMI: entre espinas mentonianas inferiores. IEMI: inferior a espinas inferiore (a, b o c dependiendo de la cantidad de forámenes inferiores).

Tabla III. Longitud y número de forámenes según posición en hueso y CBCT.

\begin{tabular}{|c|c|c|c|c|c|c|}
\hline $\begin{array}{l}\text { Longitud según } \\
\text { ubicación }\end{array}$ & $\begin{array}{c}\text { Media en mm } \\
\text { hueso }\end{array}$ & De hueso & $\begin{array}{c}\text { Número canales } \\
\text { en cada posición } \\
\text { hueso }\end{array}$ & $\begin{array}{l}\text { Media en } \\
\text { mm CBCT }\end{array}$ & $\begin{array}{c}\text { De } \\
\text { CBCT }\end{array}$ & $\begin{array}{l}\text { Número canales } \\
\text { en cada posición }\end{array}$ \\
\hline SEMS & 6,63 & 3,38 & 55 & 6,42 & 2,39 & 61 \\
\hline EEMS & 6,57 & 3,22 & 4 & 7,62 & 2,46 & 2 \\
\hline EEMSI & 5,09 & 3,10 & 17 & 6,85 & 1,7 & 15 \\
\hline EEMI & 5,56 & 3,13 & 8 & . & . & 0 \\
\hline IEMIA & 5,61 & 2,97 & 51 & 6,01 & 2,02 & 61 \\
\hline IEMIB & 4,75 & 2,75 & 14 & 4,63 & 2,44 & 28 \\
\hline IEMIC & 5,50 & . & 1 & 3,02 & 1,09 & 3 \\
\hline
\end{tabular}

SEMS: superior a espinas mentonianas superiores. EEMS: entre espinas mentonianas superiores. EEMSI: entre espinas mentonianas superiores e inferiores. EEMI: entre espinas mentonianas inferiores. IEMI: inferior a espinas inferiores (a, b o c dependiendo de la cantidad de forámenes. DE: Desviación estándar.

Tabla IV. Medidas de correlación entre números de forámenes, áreas y longitudes según posición.

\begin{tabular}{lccc}
\hline \multicolumn{1}{c}{ Variable } & $\begin{array}{c}\text { Hueso seco Media } \\
(\mathrm{DE}) \dagger \text { Mediana(RIC) }\end{array}$ & $\begin{array}{c}\text { Imagenología Media(DE) } \dagger \\
\text { Mediana(RIC) }\end{array}$ & $\begin{array}{c}\text { Correlación } \\
\text { Número de forámenes SEMS }\end{array}$ \\
Número de forámenes EEMSI & $0(0,5) \dagger$ & $1(0) \dagger$ & $0,423^{*}$ \\
Número de forámenes IEMI & $1(0,5) \dagger$ & $0(0) \dagger$ & $0,16^{*}$ \\
Área de forámenes SEMS & $0,47(0,33)$ & $1(0) \dagger$ & $0,31^{* *}$ \\
Área de forámenes EEMSI & $0,44(0,37)$ & $0,96(0,76)$ & $0,059^{* *}$ \\
Área de forámenes IEMIa & $0,53(0,43)$ & $1,01(1,09)$ & $0,342^{* *}$ \\
Longitud de canales SEMS & $6,63(3,38)$ & $0,87(0,61)$ & $0,144^{* *}$ \\
Longitud de canales EEMSI & $5,09(3,1)$ & $6,42(2,39)$ & $0,499^{* *}$ \\
Longitud de canales IEMIa & $5,61(2,97)$ & $6,85(1,7)$ & 0 \\
\hline
\end{tabular}

* Kappa. ** Correlación intraclase. DE: desviación estándar. RIC: rango intercuartílico.

\section{DISCUSIÓN}

Los resultados obtenidos en este estudio sobre población chilena muestran una similitud en el comportamiento en cuanto a la presencia y distribución en la ubicación de los forámenes linguales respecto a otras poblaciones, siendo encontrados principalmente a nivel de la línea mediana, por sobre, entre o inferior a las espinas mentonianas (Sekerci et al. 2014; Wang et al. 2015). 
La gran mayoría de las publicaciones muestra una alta prevalencia de estos forámenes en CBCT, siendo entre un $79 \%$ a un $100 \%$ en las muestras estudiadas (Babiuc et al. 2011; Gahleitner et al. 2001; Katakami et al.; Kawashima et al.; Yildirim et al. 2014). Afortunadamente, esta importante presencia, no se relaciona de manera directamente proporcional con la incidencia de accidentes graves, como hemorragias que puedan llegar a comprometer la vida aérea. En la literatura se exponen principalmente reportes de casos, pero con una prevalencia mucho menor a la presencia de estos forámenes (Balaguer-Marti et al. 2014; Flanagan; Isaacson; Kalpidis \& Setayesh; Kilic et al.).

Se observó en la muestra estudiada, una alta presencia de forámenes linguales, encontrando por lo menos un foramen, en el $97 \%$ de las mandíbulas secas estudiadas y en el $100 \%$ de los CBCTs analizados. Sin embargo, la confiabilidad entre las observaciones obtenidas en visión directa de hueso seco, con las mediciones obtenidas de la misma muestra en CBCT no fueron las esperadas. La menor cantidad de forámenes encontrados en hueso seco comparado con mandíbula, se pudo deber en parte, a una mayor capacidad de discriminación del CBCT. Se pudo encontrar canales intraoseos y buscar el foramen de inicio de estos canales en CBCT, mientras que en el hueso seco, algunas de estas entradas pudiesen haber estado obliteradas por barniz propio de la osteotecnia.

La variable utilizada pada caracterizar al foramen lingual fue el área de este reparo anatómico. En la totalidad de los estudios revisados, se utilizó el diámetro para medir el tamaño de los forámenes linguales, lo cual a nuestro juicio, induce a error debido a la morfología más bien ovalada de estos forámenes, existiendo una variedad de diámetros posibles, dentro de los $360^{\circ}$. Por esta razón se decidió describir la muestra en términos de área, lo cual es más representativo de la morfología de este reparo.

La mayoría de las mediciones de área y longitud obtenidas en este estudio, mostraron una tendencia a mayores diámetros y longitudes en las mediciones de CBCT comparadas con las mediciones de hueso seco. Esto se pudo deber en parte la diferente metodología de medición. Observamos que los peores resultados de confiabilidad se obtuvieron en las mediciones de áreas, sobre todo del área superior. La metodología utilizada en la medición de área directa en hueso, se basó en fotografías con un papel milimetrado de referencia, el cual, al estar ubicado en una zona curva (cara interna de la mandíbula) pudo haber sido distorsionado en su medida real (Cáceres et al.; Soto et al.). Por otro lado, poder captar ya sea en la fotografía directa en hueso seco, o en el CBCT, la entrada al foramen de manera ortográfica, es una fuente posible de errores en la medición, sobre todo, cuando las estructuras a medir son milimétricas. La longitud de los canales tampoco se correlacionó de la manera esperada, con una tendencia a mayores longitudes del canal a nivel de CBCT. La capacidad discriminación de la lima endodóntica utilizada para la medición directa en hueso es difícil de comparar con la capacidad de seguir mediante una imagen radiológica, al canal lingual estudiado.

Múltiples estudios evalúan la exactitud entre las mediciones realizadas de un objeto en particular, con las mediciones realizadas del mismo objeto en CBCT (Baumgaertel et al. 2009; Gerlach et al. 2014; Kim et al. 2010; Kosalagood et al.). Muchos de ellos muestran que las mediciones obtenidas no son idénticas al objeto original, pero con una exactitud comparable y clínicamente aceptable. Un estudio realizado por Gerlach et al. comparó las mediciones realizadas en cortes histológicos de mandíbula, con las mediciones realizadas en CBCT para la posición y diámetro del canal mandibular (Gerlach et al. 2014). Encontró diferencias significativas tanto para lo posición del canal, como para el diámetro de este. Respecto al diámetro, el CBCT mostró diámetros menores a los obtenidos con la muestra histológica, con un promedio de 0,52 $\mathrm{mm}$ (DE 0,26 mm) menos. Esto se pudo deber en parte, a la dificultad de discriminar en CBCT los límites del canal. Los autores, además del diámetro, encontraron diferencias en las mediciones de la posición del canal mandibular, respecto a los márgenes de la mandíbula, por lo que recomiendan un margen de seguridad de $0,76 \mathrm{~mm}$ en la posición del canal y para el diámetro del canal, un aumento en $0,74 \mathrm{~mm}$ sobre la medida entregada por CBCT. En otro estudio, se observó también una tendencia a subestimar las mediciones lineales por parte del CBCT, con un promedio de $0,17 \mathrm{~mm}$ de subestimación en el CBCT comparado con mediciones directas en cortes de mandíbulas frescas porcinas (Halperin-Sternfeld et al. 2014). Otro estudio menciona que es posible encontrar desviaciones de las mediciones ya sea de altura y grosor mandibular entre $>0$ a $1,99 \mathrm{~mm}$ en un $98 \%$ de las mediciones realizadas en CBCT (modelo ICAT $^{\circledR}$ ) comparadas con mediciones directas en el hueso (Maloney et al. 2011). En otros estudios se han encontrado sobrestimaciones en mediciones lineales a nivel mandibular y maxilar, de ente 0,33 $\mathrm{mm}$ hasta $3 \mathrm{~mm}$, respecto a las mediciones de altura y ancho de hueso alveolar (Chen et al. 2008; Gerlach et al. 2013; Mol \& Balasundaram 2008). Llama la atención que se ha descrito una menor confiabilidad de las mediciones en CBCT del sector anterior mandibular, comparado con el sector posterior respecto del hueso seco (Mol \& Balasundaram).

Se puede concluir, que las mediciones directas en el hueso tiene algunas complicaciones como, dificultad de definir una entrada ortográfica al foramen lingual, para la ob- 
tención de la imagen fotográfica, la presencia de residuos de barniz a la entrada de los forámenes y además, posibles distorsiones propias de la técnica de fotografía. Por otro lado, el CBCT tiene dificultades en la identificación ortográfica del foramen de entrada al canal lingual, también presenta complicaciones para identificar la ubicación del foramen respecto a las espinas mentonianas (EEMS, EEMSI, EEMI). Al ser estas espinas un reparo óseo hiperdenso, dificultan poder clasificar al foramen en cualquiera de estas posicio- nes. Además el seguimiento del canal en CBCT se hace difícil, si es que este se desvía de la línea mediana hacia la profundidad. Tendiendo en cuentas estas consideraciones y la alta prevalencia de estos forámenes, es recomendable previo a cualquier cirugía en el sector mandibular anterior, el estudio mediante CBCT. Además se recomienda mantener un margen de seguridad respecto a los forámenes linguales debido a una mínima distorsión esperable de estas estructuras.

CÁCERES, F.; PARDO, S.; CONCHA, G. \& SOTO, R. Presence and morphometry of lingual foramina and canal in Cone Beam CT of Chilean dry mandibles. Correlation with direct bone measures. Int. J. Morphol., 35(1):299-305, 2017.

SUMMARY: On several studies, there has been described foramina located in the inner face of the anterior mandible, whether on dry bone, conventional radiography and cone beam CT (CBCT). The aim of this study was compare the presence, position and anatomical characteristics of foramina and lingual canals found in CBCT of Chilean mandibles, with the results of the same sample studied with direct vision in dry bone. We studied 68 mandibles from the morphology unit of Universidad de los Andes. The number of teeth, foramina related to mental spines, position, area and length of each canal were evaluated, in dry bone and CBCT from the same sample. Presence of lingual foramen was found in $100 \%$ of CBCT and $97 \%$ of direct observation in bone. There were 150 foramina in dry bone and 170 foramina in CBCT. The greatest amount was found superior (bone: 55; CBCT: 61) and inferior (bone: 66; CBCT: 92) to the mental spines. . The reliability was not as expected, with a kappa for the number of upper foramina of 0.43 , an intraclass correlation for the number of lower foramen of 0.31. The upper foramen area had an intraclass correlation of 0,059 and an inferior correlation of 0.144 while, the length of the superior canals, had a 0.5 and inferior 0.246 of intraclass correlation. A trend was observed for higher diameters and canal lengths in the measurements performed with CBCT compared to the measurements in dry bone. Taking into account the high prevalence of these foramina, it is advisable prior to any surgery in the anterior mandible, the prior study using CBCT. In addition to maintaining a margin of safety with respect to lingual foramina.

KEY WORDS: Lingual foramina; Lingual canal; Cone beam CT; CBCT.

\section{REFERENCIAS BIBLIOGRÁFICAS}

Babiuc, I.; Ta rlungeanu, I. \& Pa una, M. Cone beam computed tomography observations of the lingual foramina and their bony canals in the median region of the mandible. Rom. J. Morphol. Embryol., 52(3):827-9, 2011.

Balaguer-Martí, J. C.; Peñarrocha-Oltra, D.; Balaguer-Martínez, J. \& Peñarrocha-Diago, M. Immediate bleeding complications in dental implants: a systematic review. Med. Oral Patol. Oral Cir. Bucal, 20(2):e231-8, 2014.

Baumgaertel, S.; Palomo, J. M.; Palomo, L. \& Hans, M. G. Reliability and accuracy of cone-beam computed tomography dental measurements. Am. J. Orthod. Dentofacial Orthop., 136(1):19-25, 2009.

Cáceres, F.; Ramírez, V. \& Soto, R. Influence of remnant teeth in the presence and morphometry of foramina and canals in relation to mental spine. Int. J. Morphol., 32(1):106-11, 2014.

Cerda, J. L. \& Villarroel, P. L. Evaluación de la concordancia inter-observador en investigación pediátrica: Coeficiente de Kappa. Rev. Chil. Pediatr., 79(1):54-8, 2008.

Cova, M.; Ukmar, M.; Bole, T.; Morra, A.; Lubin, E. \& Pozzi Mucelli, R. Evaluation of lingual vascular canals of the mandible with Computed Tomography. Radiol. Med., 106(4):391-8, 2003.

Chen, L. C.; Lundgren, T.; Hallström, H. \& Cherel, F. Comparison of different methods of assessing alveolar ridge dimensions prior to dental implant placement. J. Periodontol., 79(3):401-5, 2008.

Flanagan, D. Important arterial supply of the mandible, control of an arterial hemorrhage, and report of a hemorrhagic incident. J. Oral Implantol., 29(4):165-73, 2003.
Gahleitner, A.; Hofschneider, U.; Tepper, G.; Pretterklieber, M.; Schick, S.; Zauza, K. \& Watzek, G. Lingual vascular canals of the mandible: evaluation with dental CT. Radiology, 220(1):186-9, 2001.

Gerlach, N. L.; Ghaeminia, H.; Bronkhorst, E. M.; Bergé, S. J.; Meijer, G. J. \& Maal, T. J. Accuracy of assessing the mandibular canal on conebeam computed tomography: a validation study. J. Oral Maxillofac. Surg., 72(4):666-71, 2014.

Gerlach, N. L.; Meijer, G. J.; Borstlap, W. A.; Bronkhorst, E. M.; Bergé, S. J. \& Maal, T. J. J. Accuracy of bone surface size and cortical layer thickness measurements using cone beam computerized tomography. Clin. Oral Implants Res., 24(7):793-7, 2013.

Halperin-Sternfeld, M.; Machtei, E. E. \& Horwitz, J. Diagnostic accuracy of cone beam computed tomography for dimensional linear measurements in the mandible. Int. J. Oral Maxillofac. Implants, 29(3):593-9, 2014.

Isaacson, T. J. Sublingual hematoma formation during immediate placement of mandibular endosseous implants. J. Am. Dent. Assoc., 135(2):168$72,2004$.

Jacobs, R.; Mraiwa, N.; van Steenberghe, D.; Gijbels, F. \& Quirynen, M. Appearance, location, course, and morphology of the mandibular incisive canal: an assessment on spiral CT scan. Dentomaxillofac. Radiol., 31(5):322-7, 2002.

Kalpidis, C. D. \& Setayesh, R. M. Hemorrhaging associated with endosseous implant placement in the anterior mandible: a review of the literature. J. Periodontol., 75(5):631-45, 2004. 
Katakami, K.; Mishima, A.; Kuribayashi, A.; Shimoda, S.; Hamada, Y. \& Kobayashi, K. Anatomical characteristics of the mandibular lingual foramina observed on limited cone-beam CT images. Clin. Oral Implants Res., 20(4):386-90, 2009.

Kawashima, Y.; Sekiya, K.; Sasaki, Y.; Tsukioka, T., Muramatsu, T. \& Kaneda, T. Computed tomography findings of mandibular nutrient canals. Implant Dent., 24(4):458-63, 2015.

Kilic, E.; Doganay, S.; Ulu, M.; Çelebi, N.; Yikilmaz, A. \& Alkan, A. Determination of lingual vascular canals in the interforaminal region before implant surgery to prevent life-threatening bleeding complications. Clin. Oral Implants Res., 25(2):e90-3, 2014.

Kim, T. S.; Caruso, J. M.; Christensen, H. \& Torabinejad, M. A comparison of cone-beam computed tomography and direct measurement in the examination of the mandibular canal and adjacent structures. J. Endod., 36(7):1191-4, 2010.

Kosalagood, P.; Silkosessak, O. C.; Pittayapat, P.; Pisarnturakit, P.; Pauwels, R. \& Jacobs, R. Linear measurement accuracy of eight Cone Beam Computed Tomography Scanners. Clin. Implant Dent. Relat. Res., 17(6):1217-27, 2014.

Liang, H.; Frederiksen, N. L. \& Benson, B. W. Lingual vascular canals of the interforaminal region of the mandible: evaluation with conventional tomography. Dentomaxillofac. Radiol., 33(5):340-1, 2004.

Liang, X.; Jacobs, R. \& Lambrichts, I. An assessment on spiral CT scan of the superior and inferior genial spinal foramina and canals. Surg. Radiol. Anat., 28(1):98-104, 2006.

Lustig, J. P.; London, D.; Dor, B. L. \& Yanko, R. Ultrasound identification and quantitative measurement of blood supply to the anterior part of the mandible. Oral Surg. Oral Med. Oral Pathol. Oral Radiol. Endod., 96(5):625-9, 2003

Maloney, K.; Bastidas, J.; Freeman, K.; Olson, T. R. \& Kraut, R. A. Cone beam computed tomography and SimPlant materialize dental software versus direct measurement of the width and height of the posterior mandible: an anatomic study. J. Oral Maxillofac. Surg., 69(7):1923-9, 2011.

McDonnell, D.; Reza Nouri, M. \& Todd, M. E. The mandibular lingual foramen: a consistent arterial foramen in the middle of the mandible. $J$. Anat., 184 (Pt. 2):363-9, 1994.

Mol, A. \& Balasundaram, A. In vitro cone beam computed tomography imaging of periodontal bone. Dentomaxillofac. Radiol., 37(6):319-24, 2008.

Mozzo, P.; Procacci, C.; Tacconi, A.; Martini, P. T. \& Andreis , I. A. A new volumetric CT machine for dental imaging based on the cone-beam technique: preliminary results. Eur. Radiol., 8(9):1558-64, 1998.

Patil, S.; Matsuda, Y. \& Okano, T. Accessory mandibular foramina: a CT study of 300 cases. Surg. Radiol. Anat., 35(4):323-30, 2013.

Prieto, L.; Lamarca, R. \& Casado, A. Assessment of the reliability of clinical findings: the intraclass correlation coefficient. Med. Clin. (Barc.), 110(4):142-5, 1998.

Romanos, G. E.; Gupta, B. \& Crespi, R. Endosseous arteries in the anterior mandible: literature review. Int. J. Oral Maxillofac. Implants, 27(1):904, 2012.

Sekerci, A. E.; Sisman, Y. \& Payveren, M. A. Evaluation of location and dimensions of mandibular lingual foramina using cone-beam computed tomography. Surg. Radiol. Anat., 36(9):857-6, 2014.

Soto, R.; Cáceres, F. \& García, R. Presence and morphometry of foramina and canals in relation to mental spines. Int. J. Morphol., 30(2):417-21, 2012.

Wang, Y. M.; Ju, Y. R.; Pan, W. L. \& Chan, C. P. Evaluation of location and dimensions of mandibular lingual canals: a cone beam computed tomography study. Int. J. Oral Maxillofac. Surg., 44(9):1197-203, 2015.

Yildirim, Y. D.; Güncü, G. N.; Galindo-Moreno, P.; Velasco-Torres, M.; Juodzbalys, G.; Kubilius, M.; Gervickas, A.; Al-Hezaimi, K.; Al-Sadhan, R.; Yilmaz, H. G.; Asar, N. V.; Karabulut, E.; Wang, H. L. \& Tözüm, T. F. Evaluation of mandibular lingual foramina related to dental implant treatment with computerized tomography: a multicenter clinical study. Implant Dent., 23(1):57-63, 2014.
Zhang, W.; Skrypczak, A. \& Weltman, R. Anterior maxilla alveolar ridge dimension and morphology measurement by cone beam computerized tomography (CBCT) for immediate implant treatment planning. B. $M$. C. Oral Health, 15:65, 2015 .

\section{Dirección para correspondencia: \\ Dr. Reinaldo Soto, DDS \\ Especialista en cirugía oral \\ Profesor asociado \\ Unidad de Morfología \\ Universidad de los Andes \\ Monseñor Álvaro del Portillo 12455 \\ Las Condes \\ Santiago - CHILE}

Email: rsoto@miuandes.cl

Recibido : 28-12-2016

Aceptado: 29-12-2016 